\title{
Author Correction: Development of a source-exposure matrix for occupational exposure assessment of electromagnetic fields in the INTEROCC study
}

\author{
J. Vila ${ }^{1,2,3} \cdot$ J. D Bowman ${ }^{4} \cdot$ J. Figuerola ${ }^{1} \cdot$ D. Moriña ${ }^{1} \cdot$ L. Kincl $^{5} \cdot$ L. Richardson $^{6} \cdot$ E. Cardis $^{1,2,3}$
}

Published online: 21 March 2019

(c) Springer Nature America, Inc. 2019

Correction to: Journal of Exposure Science and Environmental Epidemiology;

https://doi.org/10.1038/jes.2016.60;

published online 09 November 2016
Corrigendum: This work was also funded by the European Commission grant 603794 (GERoNiMO project).

$\triangle$ J. Vila

javier.vila@isglobal.org

1 ISGlobal, Center for Research in Environmental Epidemiology,

Parc de Recerca Biomèdica de Barcelona, Barcelona, Spain

2 Universitat Pompeu Fabra, Barcelona, Spain

3 CIBER Epidemiología y Salud Pública, Madrid, Spain

4 National Institute for Occupational Safety and Health, Cincinnati, OH, USA

5 Oregon State University, Corvallis, OR, USA

6 University of Montreal Hospital Research Centre, Montreal, QC, Canada 